\section{Timing it right: The Measurement and Prediction of Flowering}

\author{
R. J. Summerfield \\ Faculty of Agriculture and Food, the University of Reading
}

\begin{abstract}
Although the model described here was developed from research in controlled environments, there is now considerable evidence that in can be applied to a very wide range of natural environments in several species. Multi-locational trials augmented by successional sowing and, if considered necessary, supplementary illumination in the field to increase daylength, can be used to estimate the values of the model coefficients: (1) to characterize germplasm collections and so predict flowering behaviour elsewhere; (2) for interpreting and understanding crop adaptation; and (3) for genetic analysis of photoperiod sensitivity. We do not yet know whether the model has any contribution to make to the understanding of the biochemical mechanisms of photoperiod and temperature responses, but at the very least, it should provide the basis for indicating the most appropriate environmental conditions, genotypes and physiological stage of the plants most suitable for such investigations.
\end{abstract}

Key words: prediction of flowering, modeling, annual crops

\section{INTRODUCTION}

Annual cycles of behaviour of plants and animals are strongly influenced by the environment. For example, responses to environmental cues control the timing of reproductive cycles, bud dormancy, hibernation, migration, diapause and other seasonal activities.

The limits to the growing season in temperate latitudes are determined mainly by temperature: in the winter the temperature is too cold. In the tropics growth is seldom limited by cold temperatures (except at high altitude), but it is commonly limited by lack of water since there is often a distinct dry season. In both cases the appropriate survival strategy for many plants, and especially those grown as annual crops, is to accumulate as much photosynthate as possible by exploiting most of the growing season, and then to flower, fruit and mature before adverse conditions set in.

Plants and animals seldom respond directly to these environmental changes - probably because they are not reliable. To start growing in mid-winter in response to a temporary warm spell, for example, could be disastrous. It is better to respond to a signal which more reliably times development in relation to the seasons. The most common one used in order to control seasonal behaviour is daylength, because the daylength on any date at any latitude is precisely predictable. In addition to daylength, plants also utilise accumulated temperature (e.g. day degrees above a particular base value). This ability to integrate temperature with time enables them to respond to a 'climatic' feature rather than to a 'weather' signal as would be the case if they responded immediately to a particular threshold temperature. In many cases, the primary photoperidoc response can, if necessary, be finetuned so as to adjust behaviour to significant annual variations in seasonal temperature.

There is at least some information on the photothermal flowering responses of thousands of species, albeit with a strong bias towards photoperiodism. Physiological signals that induce or inhibit flowering have been described; metabolic mutants in which the response to inductive daylengths is altered are increasingly targeted; but progress in understanding the physiological, genetic and molecular bases of induction has been slow. Intense research efforts have often focussed on a single clone or cultivar; experiments are commonly done at a single temperature; and there is still no means of assaying the quantity of stimulus (to flower or not to flower) produced in response to a single treatment.

Given these constraints, and the range and variety of flowering responses to photothermal conditions it has proved difficult to find patterns or to make generalizations that have some meaning and predictive value. I do not wish to lose sight of that range of responses and their ecological significance. Nevertheless, I shall attempt a synthesis and suggest generalizations. Indeed, practical utility demands both simplicity and a proven ability to anticipate biological events.

\section{THE TIMING OF FLOWERING AND CLIMATIC ADAPTATION}

People probably began to domesticate plants about 10,000 years ago and more or less simultaneously in at least three different regions - the Near East, northern China and Meso-America. Thus, agriculture began, and other crops were later domesticated in regions with pronounced wet and dry seasons, neither of which were particularly long. By the time 'seed agriculture' had come to replace 'colonization and gathering' it must have been clear that crops could only be grown satisfactorily during relatively short, wetter seasons.

In discussing the genetic adaptation of field crops to the variable and frequently stressful conditions under which they are grown, the key aim is to optimise productivity by matching the ontogeny [sequence of developmental stages] to the weather resources of the environment [e.g. duration of 
favourable temperature or water supply] and, where unfavourable extremes are unavoidable, to minimize their coincidence with more vulnerable stages. Not surprisingly, therefore, phenology [the influence of environment on ontogeny] is the most important single factor influencing genotypic adaptation.

Flowering is a particularly important event in crop development since it is a phase which is especially vulnerable to environmental stress. Furthermore it is the timing of this stage plant development that is very largely responsible for determining when cereal, pulse, oilseed crops and many vegetables will subsequently be ripe for harvest. It was 'fairly certain' about fifty years that a given genotype of any annual crop has its own definite optimal requirements of temperature and light [photoperiod] without which it cannot proceed at an economically desirable rate to flower formation, flowering and the production of seeds. Later, the photothermal control of the timing of flowering came to be recognized as 'especially important in crop adaptation'. Since then, however, successive attempts to correlate the timing of flowering in a wide range of crops with various weather variables have tended to confirm the viewpoint that the problem of flowering is a very complicated phenomenon, and there is little to be gained by considering it simple. Indeed, because the reliable prediction of flowering in fluctuating field environments has proved so difficult, a common viewpoint has been that photothermal effects are strongly curvilinear and interactive. However, I now believe that the responses are in fact amenable to relatively simple analysis to provide predictive models which are valuable in breeding, agronomy and genetics.

\section{THE ECOGEOGRAPHY OF PHOTOTHERMAL RESPONSES}

Because the earth's axis is inclined at $23.5^{\circ}$ towards the plane of the earth's orbit around the sun, there is a regular annual variation in daylength in any part of the world, although the extent of that variation depends on latitude (Table 1).

Table 1

Variation in daylength (h:min) with latitude

\begin{tabular}{|c|c|c|c|c|}
\hline \multirow{2}{*}{\multicolumn{2}{|c|}{$\begin{array}{c}\text { Latitude }\left({ }^{\circ} \mathrm{N} \text { or }{ }^{\circ} \mathrm{S}\right) \text { with } \\
\text { exemplar locations }\end{array}$}} & \multicolumn{3}{|c|}{$\begin{array}{c}\text { Daylength inclusive of Civil } \\
\text { Twilight (h:min)* }\end{array}$} \\
\hline & & \multirow{2}{*}{$\begin{array}{r}\text { Longest } \\
12: 50 \\
\end{array}$} & \multirow{2}{*}{$\begin{array}{r}\text { Shortest } \\
12: 50 \\
\end{array}$} & \multirow{2}{*}{$\begin{array}{r}\begin{array}{r}\text { Annual } \\
\text { variation }\end{array} \\
0: 00 \\
\end{array}$} \\
\hline 0 & Entebbe, Uganda & & & \\
\hline 10 & Costa Rica & $13: 29$ & $12: 19$ & $1: 10$ \\
\hline 20 & Hawaii & $14: 11$ & $11: 44$ & $2: 27$ \\
\hline 30 & Cairo & $15: 01$ & 11:04 & $3: 57$ \\
\hline 40 & Philadelphia, USA & 16:01 & $10: 22$ & $5: 39$ \\
\hline 50 & South Cornwall, UK & $17: 53$ & $9: 20$ & $8: 33$ \\
\hline 60 & Shetland Isles & $22: 25$ & $7: 48$ & $14: 37$ \\
\hline 70 & North Alaska & $24: 00$ & 0:00 & $24: 00$ \\
\hline
\end{tabular}

Given the relatively small seasonal differences in tropical daylengths it is not surprising that some of the most sensitive flowering responses to photoperiod are found in tropical species: differences of 1-020 min day ${ }^{-1}$ can be critical in rice (Oryza sativa) and cowpea (Vigna unguiculata).

Current knowledge indicates that all crops of tropical or subtropical origin are essentially short-day plants (SDPs), whereas the vast majority of crops of Mediterranean or temperate origin are long-day plants (LDPs). The only exceptions to this generalization of which I am aware are the SDPs sunflower (Helianthus annuus) and soyabean (Glycine max) which originated between $30^{\circ}$ and $35^{\circ} \mathrm{N}$ in the USA and between $34^{\circ}$ and $40^{\circ} \mathrm{N}$ in China, respectively. The exception of soyabean can be explained by a major perturbation of the intertropical convergence zone (ITCZ) in summer in north-east China which results in a late growing season.

Many species of crops also include genotypes which are insensitive to photoperiod, i.e. day neutral plants (DNPs), but these are often the product of special selection - either deliberate, or as an inevitable consequence of adaptation to regions outside the latitude of origin. For example, given that tropical plants typically have short-day responses flowering would be excessively delayed in the long days of temperate summers unless, in the course of their spread from the low latitudes, strains were selected which were much less sensitive or insensitive to photoperiod. Conversely, species of temperate origin with long-day responses could not adapt to the short days of the tropics unless strains with little or no sensitivity were selected. Thus the rice landraces of tropical origin (Oryza stava spp. indica) are typically very sensitive SDPs while those adapted to the temperate latitudes of Japan, Europe and USA (spp. japonica) are typically much less sensitive or insensitive. Examples from vegetables are not always so clear but tomato (Lycopersicon esculentum), for example, was probably domesticated in Central America and is essentially a short-day species but those cultivars in commercial production tend to be relatively insensitive to daylength, probably as a results of intensive breeding in temperate latitudes. The same applies to common bean (Phasseolus vulgaris). On the other hand the photoperiod sensitivity of lentil (Lens culinaris) which is a long-day species originating in West Asia is less in tropical landraces. The same applies for wheat (Triticum aestivum).

Another feature in the control of flowering is that many LDP species include genotypes in which flowering is advanced by cool temperatures - a response described as vernalization. Such a response appears to be extremely rare amongst SDPs, and in fact, only one confirmed example is known, namely Chrysanthemum moriflorum, which originated in China between 20 and $35^{\circ} \mathrm{N}$.

It is plausible that SDPs are typical of the tropics because the growing season is limited by rain (not by temperature or irradiance) and that the rainy season is a product of the ITCZ. This zone tends to coincide 
with the latitude where the sun is overhead at midday, and the sun's zenith moves from $23.5^{\circ} \mathrm{N}$ in June to $23.5^{\circ} \mathrm{S}$ in December. Crops ideally ripen towards the end of the rainy season after the accumulation of sufficient vegetative growth, and when the conditions are becoming drier and more suitable for the maturation of seeds. In these circumstances only a short-day response could results in appropriate phenology.

In Mediterranean and temperate latitudes it is also and advantage for crops to ripen towards the end of the growing season, and in a warm and not unduly wet season for seed maturation. The growing season in these regions is primarily limited by cool temperatures and, to a lesser extent, by low irradiance during the winter. In Mediterranean climates it is also curtailed by summer drought. Accordingly most crops flower in the spring in the Mediterranean and in the early summer in temperate latitudes - in both cases when the days are lengthening. Under these circumstances, only a longday response can ensure appropriate phenology; and in the case of autumn-sown annuals or biennials, this long-day response needs to be supplemented by a prior vernalization requirement in order to prevent the plants responding to the relatively long days of autumn.

In spite of the fact that plant photoperiodism was discovered more than 80 years ago and has been widely researched since then, the mechanisms at the molecular and biochemical level are still largely a mystery. Indeed, flowering in LDPs is not a simple mirror image of the SDP system; unlike SDPs, where night length is of overriding importance, light quality and quantity during the photoperiod are of considerable importance in LDPs. In both responses we know that the phytochrome pigment is involved. There is evidence too for substances which can be transmitted form leaves which either promote or inhibit flowering, but nothing is known of their structure or properties. In terms of fundamental understanding, animal photoperiodism fares no better.

\section{A QUANTITATIVE MODEL TO PREDICT FLOWERING}

Luckily, it is not necessary to understand the underlying mechanisms in order to quantify and predict the flowering responses of crops. Indeed, there is no guarantee that a knowledge of the molecular events would help to predict and quantify the overall response. [In passing, just think of the enormous amount of detail which is known about the molecular and biochemical mechanisms of photosynthesis, so fundamental to plant growth, yet none of this knowledge has so far helped in crop improvement.]

In developing a quantitative flowering prediction model my research colleagues and I (see Acknowledgements) began from the concept that most responses to temperature in chemistry or physiology are best considered as rates. In the case of flowering, we are concerned with the rate at which the plant develops through the vegetative phase until a meristem changes its anatomy and function to become reproductive (flower initiation) or until mature flowers are visible (flowering). That rate cannot be measured directly but, like an enzyme reaction rate, it can be inferred by taking the reciprocal of the time taken for the end-point to be reached. So, although we record the time in days taken from sowing to flowering $(f)$, and this is what we are ultimately concerned to predict, I concentrate here on the more fundamental property of rate of progress towards this event $(\mathrm{l} / \mathrm{f})$.

The main advantages of this approach are as follows:

1. Typically the separate responses to both photoperiod and temperature become linear over wide ranges of conditions if phenology data are transformed to rates.

2. Whereas large, significant interactions occur between temperature and photoperiod responses if data are analyzed as time to flowering, these interactions often disappear if rates are used.

3. A consequence of (1) and (2) is that simple equations without interaction terms within certain well-defined limits can be developed to predict rates of progress towards flowering and therefore, indirectly, the times taken from sowing to flowering.

4. As there are no interaction terms in such equations if it possible to identify and measure the separate genetic control of photoperiodsensitivity and of temperature-sensitivity.

5. The recognition that linearized rate equations may be applied to the data permits the application of the concept of thermal time (for photoperiodinsensitive genotypes) or an analogous concept of photothermal time (for photoperiod-sensitive genotypes). The use of these concepts allows the prediction of times to flowering in environments which are not constant, e.g. in natural environments where temperature fluctuates and photoperiod changes systematically. It is not always realized that the concepts of thermal time and photothermal time are only legitimate if rates of development are linearly related to temperature and/or photoperiod.

6. As a consequence of (3) and (4), the genotypically controlled values of the parameters which describe sensitivity to photoperiod and temperature can, where appropriate models have been developed, be estimated from responses in only a few controlled, but carefully chosen, environments (in some cases, in theory, no more than four may be essential). As a consequence of (5), it is possible to use fluctuating field conditions, or to integrate the use of controlled and natural environments to estimate these parameters.

7. A consequence of (5) and (6) is that simple and economic techniques may prove feasible for screening large germplasm collections for 
sensitivity to photoperiod and temperature in a way which allows rational genetic analysis and predictions of time to flowering in a wide range of environments.

The experimental results and arguments which led to these conclusions are described in detail elsewhere (see References, Figure 1). Only the principal features of the photothermal models are summarized here (Box 1) and illustrated for soyabean in Figures $1 a, b$.
The three intersecting planes described by equations 1,2 and 3 , which meet at the boundaries defined by equations 4,5 and 6 , form the triple-plane rate model of development which has now been shown to be applicable to a very wide range of species, e.g. cowpea; soyabean; mung bean (Vigna radiata) and related Vigna spp.; Bambara groundnut (Vigna subterranea); common bean; faba bean (Vicia faba); pea (Pisum sativum); chickpea (Cicer arietinum); subterranean clover (Trifolium subterraneum); barley; wheat; rice and lentil.

Box 1: The general triple-plane rate mode of flowering response to the photothermal environment

(as illustrated in Figure 1a, b)

There are three basic equations (1, 2 and 3 below) which together define the time taken to flower in any environment (except in supraoptimal temperatures or when other stresses are severe). The equations involve six genotypic coefficients ( $\left.a, b, a^{\prime}, b^{\prime}, c^{\prime}, d^{\prime}\right)$ all of which have identifiable biological significance and have values which are independent of the environment.

In photoperiod-insensitive plants (Figure 1a), or in daylengths shorter than the critical daylength $\mathrm{P}_{\mathrm{c}}$ in short day plants (plane B in Fig. 1b) or in daylengths longer than $\mathrm{P}_{\mathrm{c}}$ in long day plants:

$$
1 / f=a+b T
$$

where $f$ is the time from sowing to first flower (days) and $\mathrm{T}$ is mean temperature $\left({ }^{\circ} \mathrm{C}\right.$ ).

Between the critical and ceiling photoperiods (defined below) the relation in photoperiod-sensitive genotypes is:

$$
1 / f=a^{\prime}+b^{\prime} T+c^{\prime} P
$$

where $\mathrm{P}$ is mean photoperiod $\left(\mathrm{hd}^{-1}\right)$ and where $c^{\prime}$ has a positive value in long day plants but is negative in short day plants (plane $\mathrm{C}$ in Figure 1b).

The maximum delay in flowering is reached at the ceiling photoperiod, $\mathrm{P}_{\mathrm{ce}}$ above which in short day plants, or below which in long day plants there is no further delay in flowering. In short day plants, at least, when $\mathrm{P} \geq \mathrm{P}_{\text {ce }}$ variations in either $\mathrm{P}$ or $\mathrm{T}$ do not affect $f$, and so (plane D in Figure 1b):

$$
1 / f=d^{\prime}
$$

The critical and ceiling photoperiods mark boundaries between the three planes (Figure $1 b)$ and are given by:

$$
\begin{aligned}
& P_{c}=\left[a-a^{\prime}+T\left(b-b^{\prime}\right)\right] / c^{\prime} \\
& P_{c e}=\left[d^{\prime}-\left(a^{\prime}+b^{\prime} T\right)\right] / c^{\prime}
\end{aligned}
$$

and by

and two of the temperature-determined boundaries to the planes are given by:

$$
T_{p}=\left(-a+d^{\prime}\right) / b
$$

where $T_{p}$ is the temperature above which photoperiod-sensitivity genes are expressed, and by:

$$
T_{b}=-a / b
$$

where $T_{b}$ is the base temperature below which it is too cool for progress to flowering to occur
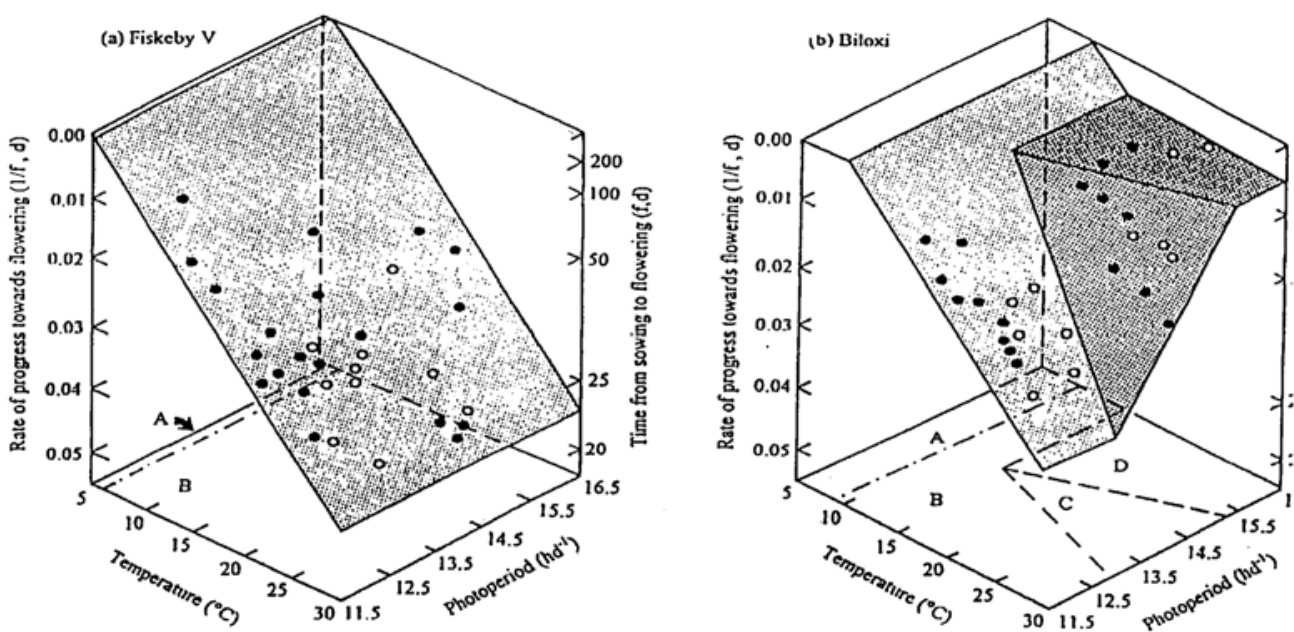

Figure 1: Photothermal flowering responses for two genotypes (a) Fiskeby V and (b) Biloxi of the SDP soyabean, detemrnide from observations from crops sown on various dates at six sies in Australia in 1986-1988 (•) and at one site in Australia and two in Taiwan in 1989-1990 (O). The vertical scale (1f) on the left hand ordinate is converted to $f$ as a non-linear scale on the right of each figure. The base of each graph has been divided into up to four sectors by vertically projecting: (A) the domain of environments where flowering cannot occur $(1 / f=0)$, because the tempereature is too cool; (B) where the time taken to flower depends on temperature only; $(\mathrm{C})$ the domain of environments where both photoperiod and temperature independently control the time taken to flower; and (D) the domain of environments in which photoperiodic delay is maximal and where variations in photoperiod or temperatures have no effect. the line between sectors B and C represents the critical photoperiod and that between C and D represents the ceiling photoperiod (From Summerfield et al., 1993). 
In some cases only one of the three planes of the model is required, e.g. only the thermal plane (equation 1) is needed when dealing with photoperiod-insensitive plants at suboptimal temperatures. In other cases in photoperiod-sensitive plants it may be that the range of relevant natural environments falls mostly within the photothermal plane (equation 2), as has been found when applying the model to lentil.

In some cases, however, the range of conditions which have to be considered spreads over two or three of the planes, and when dealing with multilocation sowing date trials there is a potential difficulty of deciding which observations near a boundary should be allocated to each plane on either side of it. However, the problem has been solved statistically and operationally by the development of an iterative computer procedure called RoDMod. Examples of applying this to field data for soyabean are shown in Figure $1 b$.

Figure $1 b$ demonstrates that the photothermal environment can be divided into different domains according to their influence on development. In two of these planes (the thermal and photothermal) there is ample evidence that the response to temperature is linear; whereas in the other (the plane of maximum delay) there is no response in soyabean. There is also considerable evidence that the rate of progress of a number of developmental processes is a linear function of temperature. The response has been examined in greatest detail for seed germination. It is by no means clear why the response of developmental rate to temperature between the base and optimum values should be linear, even though there has been some speculation. It is even less clear why the rate of progress towards flowering should be a linear function of photoperiod between the critical and ceiling values. But it is a fortunate circumstance that reponse to both temperature and photoperiod within any domain are linear and without interaction since this simplified the mathermatics. If the raw data, i.e. times from sowing to first flower, are not transformed to rate then clearly powerful interactions occur. The transformation to rates removes the need for an interaction term and also has another statistical advantage: times to flower show non-homogeneous variance - the variance increasing with delay in flowering - a common observation of experimentalists. Transformation to rates removes this problem. Not only does the use of rates legitimize the statistical analysis of responses but it also takes the investigation nearer to the underlying physiology, for it is difficult to avoid the deduction that the time taken to reach an endpoint such as flowering depends on the rate of the processes leading to it. The converse would be illogical.

Although these analytical and theoretical advantages of using a model based on rates are important, the main virtue of the triple-plane rate model is that it involves relatively few coefficients $\left(a, b, a^{\prime}, b^{\prime}, c^{\prime}, d^{\prime}\right)$, all of which, and their derivatives, have clearly defined biological meaning (e.g. $c$ ' indicates relative photoperiod sensitivity; $a / b$ is the base temperature) and, of paramount importance, the six coefficients are not affected by the environment but are genotypic characters which determine phenotypic response to the environment, and which can predict this response quantitatively.

Although it is possible to investigate the genetics of photoperiodism without a knowledge of the quantitative nature of the photoperiod and temperature responses outlined here, the results could be - and in some cases have been - misleading.

For example, if photoperiod sensitivity were to be assessed based on the difference in time to flowering in two contrasting daylengths, the value obtained would depend not only on temperature but also on whether the two photoperiods selected are within environmental domain B or one observation is in domain A or C. Even more problems of interpretation can arise, as they often did in earlier work, if comparisons are made on the basis of two photoperiodic regimes in which the number of plants which have flowered after an arbitrary time are recorded, rather than measuring the time taken for each sample to flower, from which rates can be derived. Furthermore, since temperature as well as photopeeriod affects the flowering responses, it is important to compare responses at different temperatures to determine whether or not a gene which influences photoperiod sensitivity also affects the response, or is affected by the response, to temperature.

In the soyabean genome five so-called maturity loci have been identified with two alternative alleles at each locus, viz. $E_{1} / e_{1}, E_{2} / e_{2}, E_{3} / e_{3}, E_{4} / e_{4}$ and $E_{5} / e_{5}$. We have investigated the effects of all eight possible isolines of three of these loci $E_{1} / e_{1}, E_{2} / e_{2}$, and $E_{3} / e_{3}$ in a cv. Clark background. (cv. Clark has the following complement of maturity genes: $e_{1} E_{2} E_{3} E_{4} e_{5}$.) It was shown that none of the genes affects temperature sensitivity ( $b$ or $b^{\prime}$ ) and that the major effect of the dominant alleles is on photoperiod sensitivity, e.e. on $c$ ', although additional (pleiotropic?) effects can also be recognized. There were three categories of increasing sensitivity to photoperiod: (1) least sensitivity (but nevertheless still slightly sensitive, probably due to the presence of $E_{4}$ in the cv. Clark background) represented by $e_{1} e_{2} e_{3}, e_{1} E_{2} e_{3}$ and $e_{1} e_{2}$ $E_{3}$; (2) intermediate sensitivity represented by $E_{1} e_{2}$ $e_{3}$ and $e_{1} E_{2} E_{3}$; and (3) most sensitivity represented by $E_{1} E_{2} e_{3}, E_{1} e_{2} E_{3}$ and $E_{1} E_{2} E_{3}$. Thus, photoperiod sensitivity is dominant. Increase in sensitivity never accelerates flowering, but delays it in any photoperiod greater than the critical photoperiod. $E_{1}$ has the greatest effect. While neither $E_{2}$ nor $E_{3}$ have any effect independently, together they show epistasis and produce roughly the same effect as $E_{1}$ on its own. Furthermore, either $E_{2}$ or $E_{3}$ can enhance the effect of $E_{1}$

With the increase in photoperiod sensitivity induced by these gene combinations, there is also concommitant decrease in the value of $d$, i.e. an increase in the time taken to flower in unfavourable photoperiods greater than the ceiling value. The maximum time taken to flower (at a mean 
temperature of $25^{\circ} \mathrm{C}$ ) in these isolines varies from about 50 days in the least sensitive genotypes to about 100 days in the most sensitive one.

All these effects of the E-maturity genes which result in different phenotypic responses in different environments are genetically characterized mostly by the correlated values of the $c$ ' and $d$ ' coefficients, although $a^{\prime}$ is also affected. We believe, however, that the most important effects of the genes for practical applications reside in the values of the $c$ ' and $d^{\prime}$ coefficients and, since the values are correlated, it might well prove adequate when screening germplasm to concentrate on $c^{\prime}$, thus reducing the number of environments required.

Although the model described here was developed from research in controlled environments, there is now considerable evidence that it can be applied to a very wide range of natural environments in several species. Multi-locational trials augmented by successional sowings and, if considered necessary, supplementary illumination in the field to increase daylength, can be used to estimate the values of the model coefficients: (1) to characterize germplasm collections and so predict flowering behaviour elsewhere; (2) for interpreting and understanding crop adaptation; and (3) for genetic analysis of photoperiod sensitivity. We do not yet know whether the model has any contribution to make to the understanding of the biochemical mechanisms of photoperiod and temperature responses, but at the very least, it should provide the basis for indicating the most appropriate environmental conditions, genotypes and physiological stage of the plants most suitable for such investigations.

\section{ACKNOWLEDGEMENTS}

My scientific career has been enriched by personal friendships and productive collaboration with several colleagues in UK and abroad, together with a family of more than 30 graduate students from 17 countries. I am especially indebted to the following colleagues for the exciting work we shared over the periods given in parentheses: Professors E. H. Roberts, UK (1973-98); F. J. Muehlbauer, USA (1979 to date); R. H. Ellis, UK (1984 to date); H. C. Wien, USA (1973-84); R. J. Lawn, Australia (198595); J. D. H. Keatinge, UK (1993-98) and P. Hadley, UK (1977-81); Drs F. R. Minchin, UK (1973-80); W. Esrkine, Syria (1978-98); P. Q. Craufurd, UK (1991 to date); Qi Aiming, UK (1989-98) and T. R. Wheeler, UK (1993 to date). I have as well enjoyed first class technical and engineering support from Mrs. C. Hadley, and Messrs K. E. Chivers, S. D. Gill, A. Pilgrim, D. Dickinson and the late A. C. Richardson.

REFERENCES

The scientific background of research on the photothermal regulation of flowering has been reviewed in detail elsewhere:

Roberts, E. H.-Summerfield, R. J. (1997): Measurement and prediction of flowering in annual crops. In: Atherton, J. G. (ed) Manipulation of Flowering, Butterworths, London, 17-50.

Roberts, E. H.-Summerfield, R. J.-Ellis, R. H.-Wheeler, T. R. (1997): The induction of flowering. In: Wien, H.C. (ed) The Physiology of Vegetable Crops, CAB International, Wallingford, 69-99.

Summerfield, R. J.-Roberts, E. H.-Lawn, R. J. (1991): Towards the reliable prediction of time to flowering in six annual crops. 1. the development of simple models for fluctuating field environments. Experimental Agriculture, 27, 11-31.
And for soyabean in particular, see:

Summerfield, R. J.-Lawn, R. J.-Qi, A.-Ellis, R. H.-Roberts, E. H.Chay, M. W.-Brouwer, J. B.-Rose, J. L.-Shanmugasundaram, S.-Yeates, S. J.-Sandover, S. (1993): Towards the reliable prediction of time to flowering in six annual crops. II. Soyabean (Glycine max). Experimental Agriculture, 29, 253289 . 